\title{
Missing Motherhood: Jordanian Women's Experiences with Infertility
}

\author{
Hala Mahmoud Obeidat, ${ }^{1}$ Adlah M. Hamlan, ${ }^{2}$ and Lynn Clark Callister ${ }^{3}$ \\ ${ }^{1}$ Maternal Child Health Nursing Department, Princess Muna College of Nursing, Mutah University, Amman, Jordan \\ ${ }^{2}$ Jordan University, Amman, Jordan \\ ${ }^{3}$ Brigham Young University College of Nursing, Provo, UT 84602, USA
}

Correspondence should be addressed to Lynn Clark Callister; callister-lynn@comcast.net

Received 10 January 2014; Revised 16 April 2014; Accepted 30 April 2014; Published 5 June 2014

Academic Editor: Takahiro Nemoto

Copyright (C) 2014 Hala Mahmoud Obeidat et al. This is an open access article distributed under the Creative Commons Attribution License, which permits unrestricted use, distribution, and reproduction in any medium, provided the original work is properly cited.

\begin{abstract}
Aim, Background, and Introduction. Bearing and rearing children are an important part of life in nearly all cultures and are a central role for Jordanian Muslim women. Infertility can create anxiety, stress, and depression for couples who are infertile. Women frequently bear the emotional stigma of a couple's infertility. There is a paucity of literature focusing on Jordanian Muslim women experiencing infertility and failed assistive reproductive technology. Therefore, this study explored these women's lived experience. Methods. Qualitative data were collected through interviews with 30 Jordanian Muslim women who experienced failed assistive reproductive technology for infertility. Perceptions of experiences with failed treatment of infertility were documented and analyzed. Results. Major themes were identified: missing out on motherhood and living with infertility, experiencing marital stressors, feeling social pressure, experiencing depression and disappointment, having treatment associated difficulties, appreciating support from family and friends, using coping strategies, and fear of an unknown future. Discussion, Conclusion, and Implications for Clinical Practice. Being infertile significantly influences the physical, emotional, social, and spiritual health of Jordanian Muslim women as well as their quality of life. Perceived social support and personal coping strategies were used by study participants to mediate failed attempts to conceive. Designing and implementing culturally appropriate interventions for Muslim women globally who are experiencing infertility are essential.
\end{abstract}

\section{Introduction}

Children provide their parents the existential role of participating in the continuity of the family, the culture, and the community. Most societies, especially in developing countries, are structured to rely on children for the future care and maintenance of older family members [1]. The United Nations (UN) Declaration of Human Rights recognizes that adults, without any limitations due to race, nationality, or religion, have the right to marry and have a family [2]. Infertility has been recognized by WHO as a public health issue, and the right to infertility treatment is part of Millennium Development Goal number 5 [3].

Infertility is a condition in which pregnancy has not occurred after one year of unprotected, well-timed intercourse [4]. Infertility is a reproductive health indicator, such as maternal mortality rates. It is an important global issue where infertility is perceived as a tragedy for many women who may be ostracized and stigmatized in their sociocultural environment [5].

Impaired fertility affects approximately 80 million people globally, with rates ranging from less than $5 \%$ to over 30\% [2]. The rates of infertility worldwide are estimated to be at least $15 \%$ in women of childbearing age, with an estimated 40.5 million women seeking treatment [6]. Even with the advancement in reproductive technology, the incidence of infertility is expected to increase to 7.7 million by 2025 [7]. In Jordan, the primary infertility rate is estimated to be $3.5 \%$ and the secondary infertility rate $13.5 \%$ [8]. A more recent source concludes that the prevalence of infertility in Jordan is not definitive which may be related to differing definitions of infertility [9].

Women frequently bear the emotional stigma of a couple's infertility even though infertility is shared by both men and 
women. In $35 \%$ of infertile couples the woman is infertile, in $35 \%$ the man is infertile, and in the remaining $30 \%$ the problem is either shared by both partners or of undetermined origin [4].

Islam is the second largest religion in the world, with 25\% of the global population espousing these religious beliefs and practices [10]. In Jordan, which is an Arab Muslim nation, marriage is strongly linked to procreation and family formation. Adoption is not a choice for infertile couples due to religious prohibitions. Therefore, diagnosis of infertility itself is stigmatizing and may lead to significant levels of emotional distress. Women for whom child bearing is a major object of their lives may be hesitant to reveal their infertility problems even to their closest friends and relatives. In Muslim communities, Islam encourages family formation and assisted reproduction, when indicated, within the frame of a committed legal marital relationship $[11,12]$.

\section{Significance and Background}

The medicalization of infertility may lead to a disregard of the feelings infertility may engender, including depression, anger, anxiety, emotional distress, loss of control, shame, stigmatization, and feelings of isolation [13-16]. Regardless of the cause of the infertility, women bear the burden of invasive procedures related to diagnosis and management of infertility [4]. Health care providers may view infertility as a medical condition rather than taking a holistic approach to caring for couples who are infertile.

Qualitative approaches can describe the lived experience of women who are experiencing infertility. No published studies could be found in the literature focusing on the infertility experience of Jordanian Muslim women. Such research can guide health care providers in planning and developing holistic care that addresses the cultural and psychosocial needs of women who are infertile. The aim of the study was to explore Jordanian Muslim women's lived experience of infertility.

The Biopsychosocial Theory of Infertility conceptualizes infertility as an acute life crisis with long-term implications for the individual, his or her partner, their relationship, and family and friends [17]. The stressors of infertility are categorized as psychological, physical, and interpersonal. According to this theory, social support and other coping strategies mediate the effect of infertility. This theory was used to guide data collection and analysis.

Social stressors of infertility may differ according to societal norms. In developed societies, voluntary childlessness is viewed as a more viable and legitimate option and women without children are often presumed to be voluntarily childfree. However, in developing countries like Jordan, bearing and rearing children are central to women's power and wellbeing and stigma related to infertility may be greater $[18,19]$.

The central role of motherhood for Jordanian Muslim women has been described. For example, a 39-year-old mother of seven children noted, "Our prophet Mohammed said, 'Reproduce and have children as I am in the life after. I will be proud of you in front of the nations"' [20]. In another study, a Jordanian Muslim primipara said, "People start asking after the first month of marriage whether you "save anything inside your abdomen" meaning, "are you pregnant yet?" So is the nature of life. I got pregnant after two months of marriage" [21].

According to Islamic tradition, having children is the most important Muslim religious injunction [22]. Procreation is encouraged, and a bride's status is not ensured until she demonstrates proven fertility. Pressure to have children includes cultural traditions, familial expectations, and religious injunctions. In addition to Islamic beliefs about the sacred nature of bearing children, children are highly valued for social prestige as well as the potential for future economic and physical support of their parents [23-26].

The impact of infertility on marital relationships depends on the sociocultural context. In settings where women's roles are more closely tied to having children, where producing children for one's family is considered an important obligation and where marriage is defined in terms of producing and raising children, infertility is likely to have a greater negative impact on the couple's relationship [18]. There are gender differences in response to infertility. In a study of Dutch couples experiencing infertility, women had lower levels of quality of life than their partners [27].

Social support and coping are considered to be mediators of infertility stress experience. Studies on psychological interventions for infertile couples suggest that psychosocial interventions could reduce negative effect associated with infertility [28, 29]. Women receiving assisted reproductive technologies cope with the stress of infertility by using their social support resources $[28,30]$.

One of the coping strategies that women use in their experience of infertility is making a conscious effort to create a new life that created a space to move forward by shifting from grief to focusing their energy toward the future [31]. However, coping strategies which are beneficial to one individual may be problematic for his or her partner. Couples with men using high amounts of distancing, while their partner used low amounts of distancing, reported higher levels of distress [32].

\section{Methodology}

This descriptive qualitative study has qualitative phenomenological components [33]. Such research is useful in increasing our understanding of complex life experiences such as infertility. The study participants were thirty Muslim women experiencing infertility with age range from 26 to 42 years with mean age of $32 \pm 5.2$ years. Table 1 presents the demographic characteristics of study participants. They were selected purposively by using snowball sampling technique through professional and social networking. The participants met the inclusion criteria including failure to conceive after at least one year of unprotected sex, identification of self as being infertile, having underwent failed treatment of infertility, and being willing to share their experience.

The study participants were educated with a minimal educational level of secondary school through having graduate 
TABLE 1: Demographics.

\begin{tabular}{lcc}
\hline Demographic variable & Frequency & Percentage \\
\hline Employment status & 12 & 40 \\
$\quad$ Employed & 18 & 60 \\
$\quad$ Homemaker & 20 & 66.7 \\
Education & 10 & 33.3 \\
$\quad$ Primary & & \\
$\quad$ Secondary & Mean & Standard deviation \\
$\quad$ University & 32 & \\
\hline Demographic variable & 7 & 2.1 \\
\hline Age & & \\
Years of infertility &
\end{tabular}

degrees. They had primary infertility and experienced five to eight failed assistive reproductive procedures in infertility centers in Jordan. They were married for seven to ten years with an upper middle class income and were all employed outside the home.

3.1. Data Collection and Analysis. The data were generated by using digitally recorded interviews in the home of the participants. The interview was started with the question, "Please tell me about your experience of not having children." In addition, the interview included questions based on a review of the literature, the researcher's clinical experience, and the Biopsychosocial Theory of Infertility.

Data analysis was concurrent with data collection. Data collection continued until saturation was reached. The interviews were transcribed into text by the interviewer shortly after it was conducted. Before the translation of text into English, the digitally taped interviews were compared with transcript. The confirmed transcripts were translated to English language by the researcher and then back translated into Arabic by another bilingual person. Three researchers analyzed the data separately and then compared the results.

Colaizzi's [34] method of analysis and interpretation was utilized to identify the significant statement, subthemes, and major themes. Data analysis began with the researcher rereading the participant's descriptions frequently and putting herself in their place in order to understand their concepts. Reading and rereading of transcripts were used to identify the significant statements. After that, this information was grouped in meaningful units that comprise the themes clusters (subthemes). These subthemes were grouped together to provide the major themes which were supported by the researcher citing specific excerpts from the interviews [33]. Also, the nonverbal expressions of participants were used to support the meaning of emerged significant statements. Individual data analyses were compared and consensus was reached on the final themes by the research team (Figure 1).

3.2. Trustworthiness of the Data. Data rigor in qualitative research (trustworthiness) is how accurately the study discovers and represents the participants' experience. Immersion of researcher in the data by recurrent reading of the transcript and constant comparative of significant information was used to enhance the dependability of data. Member check was used as a potential contributor to data credibility. Also, using multiple researchers on data analysis was used to ensure the data confirmability.

3.3. Ethical Consideration. The study was approved by the Ethical Committee of Jordan Royal Medical Services. Participants provided written informed consent upon recruitment as well as a process consent which was used throughout the interviews. Confidentiality and privacy were ensured.

\section{Study Findings}

Study participants were Jordanian Muslim women who had not become pregnant after at least one year of unprotected sex. They were all employed and had five to eight failed trials of assistive reproductive technology procedures.

The results are expressed in major themes that reflect the Biopsychosocial Theory assumptions. The overriding theme is missing motherhood and living with infertility. Others include experiencing marital stressors, feeling social pressure, experiencing depression and disappointment, feeling treatment associated difficulties, appreciating support from family and friends, and fearing an unknown future. Figure 2 presents the model of Biopsychosocial Theory showing related major themes emerging from study findings.

\section{Missing Motherhood and Living with Infertility}

The overriding theme of participants experiences with infertility was missing motherhood. Study participants described their dreams of having children and their pain at not being as other women who seemed to become pregnant so easily. One woman said, "Every one of us dreams of having children. I married at age 23. Everyone dreams that when she arrives in her thirties she [will have] one or two children. Every one of us dreams of being a mother." She described being a mother as "life's sinew" and said her seven years of seeking to become pregnant seemed so long.

The participants spoke with deep emotion about seeing other women pregnant, "Sometimes you see women who complain about the pregnancy: 'ooh, my abdomen is large. I am tired' and at the same time you wish to be just like them for just one day, and I couldn't. Of course this hurts, hurting my [heart] more than any other thing."

5.1. Experiencing Marital Stressors. The participants reported marital stressors such as "querying the social questioning," "lack of relation intimacy," and "feeling guilt." They described social questioning regarding their situation with the intrusion into a very personal and painful experience. One study participant said, "People around us still pressure us and still ask, 'Why?" What did you do?' When somebody talks about the issue, we start to argue [with each other]." Participants felt that infertility contributed to a lack of intimacy in the marital 


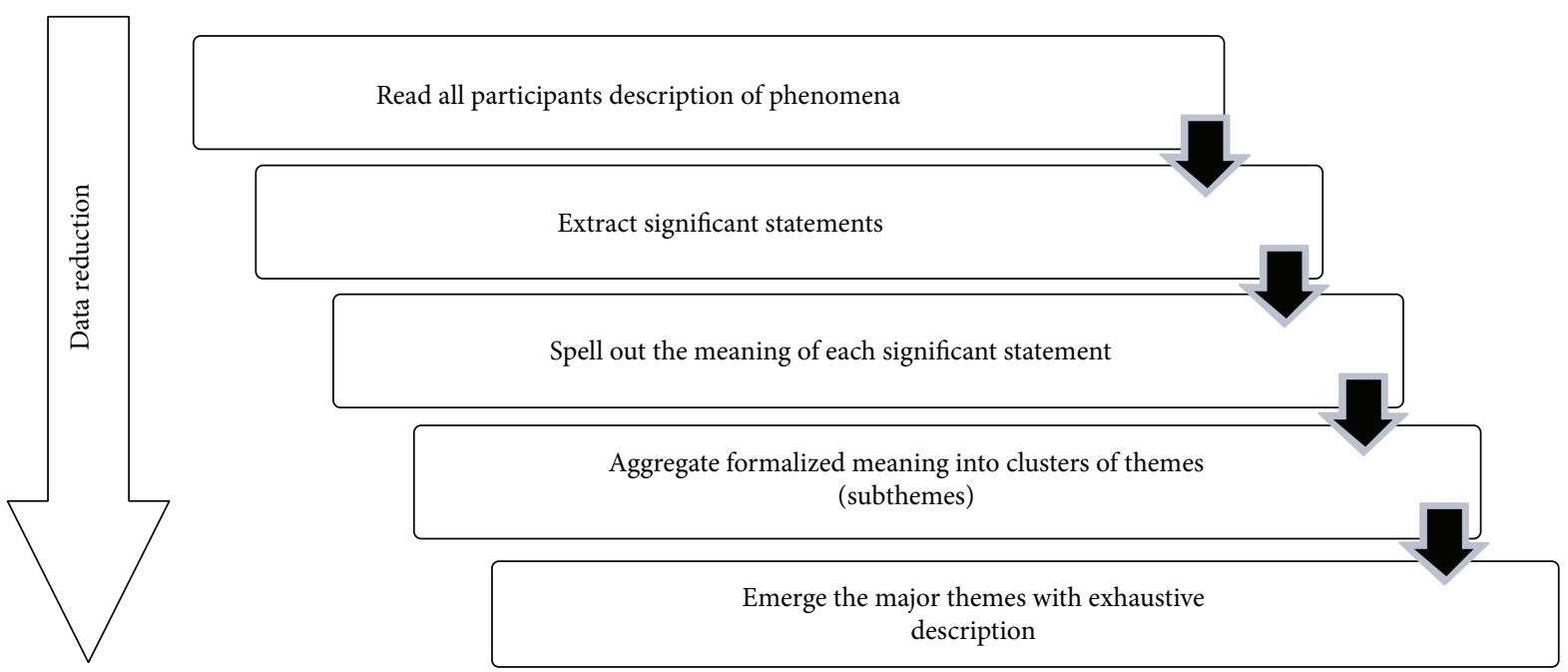

FIgURe 1: Data analysis.

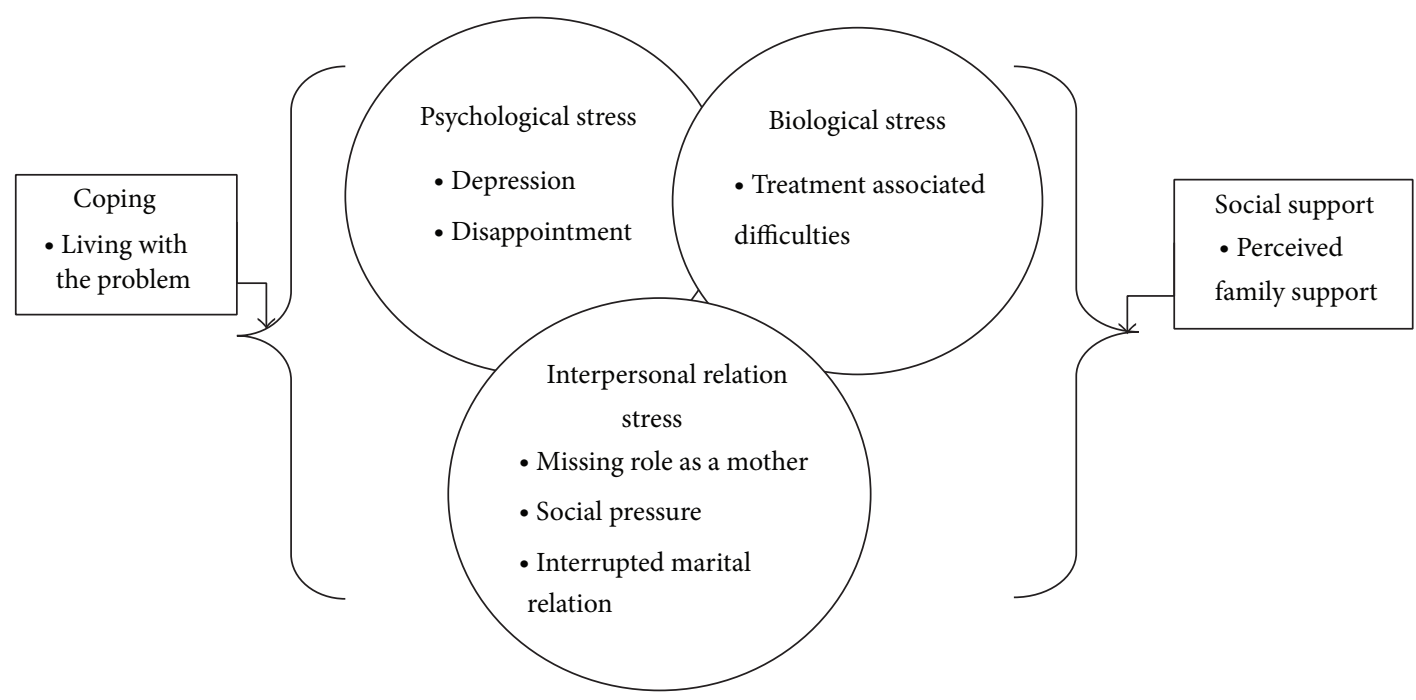

FIGURE 2: Study results as within the framework of the Biopsychosocial Theory of Infertility.

relationship since sexual relations were focused on getting pregnancy rather than as an expression of love.

Feelings of guilt emerged when they spoke of their inability to provide a child for their husbands as expressed by one wife, "You are affected when you see your husband playing with other people's children. You feel that he also has a desire to have a child. You feel guilty."

Also, feeling of failure resulted from the participant's response to treatment failure and inability to reach the optimum goal considered as a stressor that affects the marital relationship. Some study participants feared the future, expecting their husband may divorce them or marry another woman. One woman said, "even though my husband loves me, he may decide to divorce me or marry another women to reach his goal of being a father."

5.2. Feeling Social Pressure. Social pressure was described as social questioning and social stigma. Social questioning increases stress, "of course these questions started. These questions let you still think about the issue. Every time you try to forget the issue-to live with it-people are still remembering." The participants also spoke of the social stigma as if the situation were their fault when treatment did not succeed, "They said, strange-everyone succeeded but you. This is hurting me a lot because it's out of my hands."

5.3. Experiencing Depression and Disappointment. Depression was expressed as recurrent suffering and sadness. The women reported that their suffering recurred monthly with each menstrual period signaling no pregnancy again and failed treatment, "Every month when menstruation occurs-its like a moment of suffering and sorrow because this month no pregnancy occurs." After a failed insemination procedure, with again no pregnancy, they reported weeping, crying, refusing to eat, and sleeplessness in response to treatment failure. 
Study participants repeatedly asked "why do I not become pregnant?"

Study participants spoke about having feelings of uncertainty and failure, wondering whether or not treatment would be successful in conceiving, "You are nervous, upset, afraid: maybe yes, maybe no. The days and nights passed and I was thinking [only] God knows." They wanted so much to please their husband and their parents by becoming pregnant and bearing a child. They said with pain, "Again I did not reach my goal."

5.4. Having Treatment Associated Difficulties. The participants spoke of the physiological side effects of infertility treatment, "When you take injections and hormones you have tiredness, increase in heart rate, and [rapid] breathing. You feel hot because all of your hormones are disturbed."

The women described the financial burdens associated with infertility treatment, since it was not covered by health insurance, saying, "you still pay and pay and at last no results." Recurrent work leaves were required, contributing to time lost from their employment.

Infertility was ameliorated by social support and using coping strategies in order to live with infertility. Coping strategies included turning to God by intensified religious practices, exercising avoidance, and continuing to pursue having a child.

5.5. Appreciating Support from Friends and Families. Most of the participants noted they had their thoughtful and sensitive colleagues in the workplace who "comfort me and this reduces my stress." Family support, both emotional and instrumental, proved very helpful. The participants reported that their family supported them emotionally by being considerate of their feelings and by advocating for them when others asked about the situation, "When anyone talked about the issue, my father in law said, 'do not pressure them. It's enough, the pressure they already have." Instrumental support was provided by the family helping to bear the costs of the medical procedures, "My family and my husband's family were helping when we have a critical situation of no money." They expressed their gratitude that their families did not give up on helping them.

5.6. Using Coping Strategies. The women lived with the problem by turning to God, utilizing avoidance strategies, and problem focused strategies. They found their spiritual beliefs in God and the practice of religious rituals helpful in coping with infertility as well as increasing their religious faith, "It's well known that we are Muslims. Always we leave everything in God's hands. First the person should trust in God. Second is to turn to prayer, invocation, and reading the Quran." They repeated that in spite of their belief that "everything is in God's hands," they could not hide their feelings of disappointment and a sense of sadness in not becoming pregnant.

Avoidance helped the couples to escape from the constant focus on having a child, "My husband and I try to change the situation. We travel especially after any procedure or when menstruation occurs." Problem focused strategies included continuing to participate actively in assisted reproductive technologies, as well as trying complementary and alternative interventions.

\section{Discussion}

Study findings document stressors associated with infertility in Jordanian Muslim women. These women described the social stressors experienced living in a society that highly values bearing children. This finding is consistent with those of Fido and Zahid [35], who reported that childlessness resulted in social stigmatization for Kuwaiti women who are infertile, placing them at risk of serious social and emotional consequences.

Social relationships may be strained as a result of infertility. In the current study lack of relationship intimacy was reported as one of the effects of infertility related to concentrating on having sexual relation to become pregnant. Consistently, in a study of Turkish women, the respondents reported negative influence of fertility problems on the sexual relationships within couples as change in the patterns of their relationships, tension and arguments between partners [36].

The assumptions of Biopsychosocial Theory and the literature indicate that the negative psychological effects of infertility can damage an individual's self-image, especially when achieving pregnancy is failed and the individual starts regarding himself or herself as worthless as well as the generating depression and self-destructive thoughts [37]. Study findings supported theoretical assumptions with reports of depression, feelings that emerged through sadness, and recurrent suffering especially when conventional medical interventions fail.

Most of the studies reported that infertile women experienced psychopathological consequences like depression, anger, anxiety, social isolation, and feelings of guilt [14-16]. Infertile Kuwaiti women exhibited a significant higher psychopathology in the form of tension, hostility, anxiety, depression, self-blame, and suicidal ideation [35]. However, participants in the current study did not disclose self-destructive thoughts, which could be related to their religious beliefs and faith as a Muslim.

Regarding the physical stressors of infertility, Gerrity [37] stated that physical effect of infertility is more direct for women because of available medical interventions. Current study findings indicated that difficulties associated with medical treatment of infertility include physiological side effects, compounded by overwhelming financial expenses and time losses.

The literature as well as the women in this study reported that the physiological side effects of infertility treatment are one of the most challenging physical stressors women experience [38].

One of the factors mediating the pain of infertility is social support. In this case, study participants reported receiving emotional support from extended family as well as instrumental support to overcome the financial burden of infertility treatment costs. Jordanian Muslim cultural values include strong family adherence. 
Appraisal of the situation resulted in the use of different coping strategies. Findings of the current study indicate that learning to live with infertility was the major theme when assisted reproductive technology failed. Trust in God was a powerful coping strategy for these women experiencing infertility. Islamic spiritual beliefs and religious rites strengthened these women's trust in God and helped them cope with this challenge. These women also utilized problem focused strategies, seeking medical solutions for infertility. This is also reflected in a study of infertile Kuwaiti women who sought medical treatment [35].

On the other hand, some study participants used avoidance as a coping strategy after failed medical treatment. Peterson and associates [39] also reported that couples who use active avoidance coping seek relief from stress through actively avoiding problematic situations and reminders of infertility.

Bardaweel and associates [9] found among 1,031 Jordanian individuals struggling with infertility, $45 \%$ used spiritual healing and herbal medicine in addition to mainstream medical interventions. The use of complementary medicine is common in developing countries including Jordan and may be associated with strong religious beliefs.

Limitations of the Study. The study has limitations including recruitment methods used to generate a convenience sample. Participants were both educated and economically secure. The inclusion of Muslim women exclusively in this study was essential in order to identify the lived experience for those in the same cultural and religion background. The generalizability of the findings is limited but transferability to other populations of women with infertility is ensured by the trustworthiness of the data.

\section{Implications for Clinical Practice and Research}

Jordanian Muslim women experiencing infertility report this having a profound impact on all aspects of their lives. This research highlights several important implications for clinical practice with global dimensions since Muslim women live throughout the world. The findings of qualitative research can be translated into clinical practice guidelines [40]. The Low Cost In Vitro Fertilization Foundation (http://www.lowcost-ivf.org/) was established in 2006 to overcome the lack of cost-effective ART available in the developing world [41]. Such noteworthy initiatives are essential to implement if Millennium Development Goal number 5 is to be met by 2015 . This may ameliorate the financial burden of infertility treatment.

Little is known about the effectiveness of complementary and alternative methods (CAM) in treating infertility, but perhaps the use of complementary methods may help couples feel that they have attempted all treatment options [9]. Nurses need to be aware of the psychosocial and emotional impact of women's experience with infertility within their sociocultural context $[42,43]$. Thus, designing and conducting supportive programs play an important role in providing quality care for them [29]. Culturally sensitive assessment of the woman's partner, extended family, and social support network is important as a means to ascertain levels of social support that may mediate the experience's effects and enhance their sense of wellbeing [26-28].

Future research is recommended to identify the lived experience of infertility by further studying the marital dyad as a unit of analysis in order to enrich our knowledge of the social perspective of the infertility experience. Longitudinal studies measuring changes over time are also recommended [15].

\section{Conflict of Interests}

No conflict of interests has been declared by the authors.

\section{References}

[1] I. Hassanin, T. Abd-El-Raheem, and A. Shahin, "Primary infertility and health-related quality of life in Upper Egypt," International Journal of Gynecology and Obstetrics, vol. 110, no. 2, pp. 118-121, 2010.

[2] World Health Organization, Reproductive Health Indicators: Guidelines for their Generation, Interpretation and Analysis for Global Monitoring, World Health Organization, Geneva, Switzerland, 2006.

[3] United Nations, "Millennium Development Goals," 2000, http://www.un.org/millennium/declaration/ares552e.

[4] Centers for Disease Control, Outline for a National Action Plan for the Prevention, Detection and Management of Infertility, Centers for Disease Control, Atlanta, Ga, USA, 2010.

[5] L. C. Callister, "Global infertility: are we caring yet?" The American Journal of Maternal Child Nursing, vol. 35, no. 3, p. 174, 2010.

[6] M. C. Inhorn, "Right to assisted reproductive technology: overcoming infertility in low-resource countries," International Journal of Gynecology and Obstetrics, vol. 106, no. 2, pp. 172-174, 2009.

[7] M. N. Mascarenhas, S. R. Flaxman, T. Boerma, S. Vanderpoel, and G. A. Stevens, "National, regional and global trends in infertility prevalence since 1990. Health surveys," PLoS Medicine, vol. 9, no. 12, Article ID e 1001356.

[8] S. Rutstein and I. Shah, Infecundity, Infertility, and Childlessness in Developing Countries, DHS Comparative Reports No. 9, ORC Macro and the World Health Organization, Calverton, Md, USA, 2004.

[9] S. K. Bardaweel, M. Shehadeh, G. A. R. Y. Suaifan, and M. V. Z. Kilani, "Complementary and alternative medicine utilization by a sample of infertile couples in Jordan for infertility treatment: clinics-based survey," BMC Complementary and Alternative Medicine, vol. 13, article 35, 2013.

[10] M. C. Inhorn and G. I. Serour, "Islam, medicine, and ArabMuslim refugee health in America after 9/11," The Lancet, vol. 378, no. 9794, pp. 935-943, 2011.

[11] M. C. Inhorn, "Making Muslim babies: IVF and gamete donation in Sunni versus shi'a Islam," Culture and Medicine and Psychiatry, vol. 30, no. 4, pp. 427-450, 2006.

[12] G. Serour, "Islamic perspectives in human reproduction," Reproductive BioMedicine, vol. 17, no. 3, pp. 34-38, 2008. 
[13] T. Cousineau and A. Domar, "Psychological impact of infertility," Best Practice and Research: Clinical Obstetrics and Gynaecology, vol. 21, no. 2, pp. 293-308, 2007.

[14] A. Galhardo, M. Cunha, and J. Pinto-Gouveia, "Psychological aspects in couples with infertility," Sexologies, vol. 20, no. 4, pp. 224-228, 2011.

[15] B. D. Peterson, C. S. Sejback, L. Pieritana, and L. Schmidt, "Are severe depressive symptoms associated with infertility-related distress in individuals and their partners?" Human Reproduction, vol. 29, no. 1, pp. 76-82, 2014.

[16] L. Schmidt, "Infertility and assisted reproduction in Denmark: epidemiology and psychosocial consequences," Danish Medical Bulletin, vol. 53, no. 4, pp. 390-417, 2006.

[17] L. A. Pasch and C. Dunkel-Schetter, "Fertility problems: complex issues faced by women and couples," in Health Care for Women: Psychological, Social, and Behavioral Influences, S. J. Gallant, G. P. Keita, and R. Royak-Schaler, Eds., American Psychological Association, Washington, DC, USA, 1997.

[18] A. Greil, K. Slauson-Blevins, and J. McQuillan, "The experience of infertility: a review of recent literature," Sociology of Health and Illness, vol. 32, no. 1, pp. 140-162, 2010.

[19] P. Jennings, "God had something else in mind: family, religion, and infertility," Journal of Contemporary Ethnography, vol. 39, no. 2, pp. 215-237, 2010.

[20] I. A. Khalaf, F. Abu-Moghli, L. C. Callister, and R. Rasheed, "Jordanian women's experiences with the use of traditional family planning," Health Care for Women International, vol. 29, no. 5, pp. 527-538, 2008.

[21] I. Khalaf and L. C. Callister, "Cultural meanings of childbirth: muslim women living in Jordan," Journal of Holistic Nursing, vol. 15, no. 4, pp. 373-388, 1997.

[22] A. Y. Ali, An English Interpretation of the Holy Quran, Ashraf Printing, Lahore, Pakistan, 1992.

[23] M. Farsoun, N. Khoury, and C. Underwood, In Their Own Words: A Qualitative Study of Family Planning in Jordan, IEC Field Report no. 6, John Hopkins University Center for Communication Programs, Baltimore, Md, USA, 1996.

[24] S. A. Kridli and K. Libbus, "Contraception in Jordan: a cultural and religious perspective," International Nursing Review, vol. 48, no. 3, pp. 144-151, 2001.

[25] S. A. Kridli, "Health beliefs and practices among Arab women," The American Journal of Maternal Child Nursing, vol. 27, no. 3, pp. 178-182, 2002.

[26] J. Schmid, S. Kirchengast, E. Vytiska-Binstorfer, and J. Huber, "Infertility caused by PCOS-Health-related quality of life among Austrian and Moslem immigrant women in Austria," Human Reproduction, vol. 19, no. 10, pp. 2251-2257, 2004.

[27] A. S. Huppelschoten, A. J. C. M. van Dongen, C. M. Verhaak, J. M. J. Smeenk, J. A. M. Kremer, and W. L. D. M. Nelen, "Differences in quality of life and emotional status between infertile women and their partners," Human Reproduction, vol. 28, no. 8, pp. 2168-2176, 2013.

[28] M. V. Martins, B. D. Peterson, V. Almeida, L. Mesquita-Guimaraes, and M. C. Costa, "Dyadic dynamics of perceived social support in couples facing infertility," Human Reproduction, vol. 29, no. 1, pp. 85-89, 2014.

[29] T. Wischmann, "Implications of psychosocial support in infertility a critical appraisal," Journal of Psychosomatic Obstetrics and Gynecology, vol. 29, no. 2, pp. 83-90, 2008.

[30] D. Gibson and J. Myers, “The effect of social coping resources and growth-fostering relationships on infertility stress in women," Journal of Mental Health Counseling, vol. 1, pp. 1-6, 2005.

[31] P. McCarthy, "Women's lived experience of infertility after unsuccessful medical intervention," Journal of Midwifery and Women's Health, vol. 53, no. 4, pp. 319-324, 2008.

[32] B. Peterson, C. Newton, K. Rosen, and R. Schulman, "Coping processes of couples experiencing infertility," Family Relations, vol. 55, no. 2, pp. 227-239, 2006.

[33] H. S. Speziale, H. J. Streubert, and D. Carpenter, Qualitative Research in Nursing, Lippincott Williams \& Wilkins, Philadelphia, Pa, USA, 2011.

[34] P. Colaizzi, Reflection and Research in Psychology, Kendall Kent, Dubque, IL, USA, 1973.

[35] A. Fido and M. A. Zahid, "Coping with infertility among Kuwaiti women: cultural perspectives," International Journal of Social Psychiatry, vol. 50, no. 4, pp. 294-300, 2004.

[36] F. B. van Rooij, F. van Balen, and J. Hermanns, “The experiences of involuntarily childless Turkish immigrants in the Netherlands," Qualitative Health Research, vol. 19, no. 5, pp. 621-632, 2009.

[37] D. Gerrity, "A biopsychosocial theory of infertility," The Family Journal, vol. 9, no. 2, pp. 151-158, 2001.

[38] V. Madge, "Infertility, women and assisted reproductive technologies: an exploratory study in Pune, India," Indian Journal of Gender Studies, vol. 18, no. 1, pp. 1-26, 2011.

[39] B. Peterson, M. Pirritano, J. Block, and L. Schmidt, "Marital benefit and coping strategies in men and women undergoing unsuccessful fertility treatments over a 5-year period," Fertility and Sterility, vol. 95, no. 5, pp. 1759-1763, 2011.

[40] P. S. Hinds, J. S. Gattuso, E. Barnwell et al., "Translating psychosocial research findings into practice guidelines," Journal of Nursing Administration, vol. 33, no. 7-8, pp. 397-403, 2003.

[41] I. D. Cooke, L. Gianaroli, O. Hovatta et al., "Affordable ART and the Third World: difficulties to overcome," Human Reproduction, vol. 10, no. 1, pp. 93-96, 2008.

[42] H. Azaizeh, B. Saad, E. Cooper, and O. Said, “Traditional Arabic and Islamic medicine: a re-emerging health aid," Evidence-based Complementary and Alternative Medicine, vol. 7, no. 4, pp. 419424, 2010.

[43] A. Oweis, "The Hashemite Kingdom of Jordan," in Cultural Health Assessment, A. Polyanskaya, Ed., Elsevier, New York, NY, USA, 4th edition, 2008. 


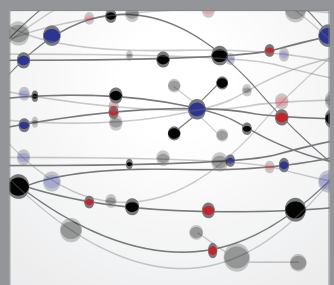

The Scientific World Journal
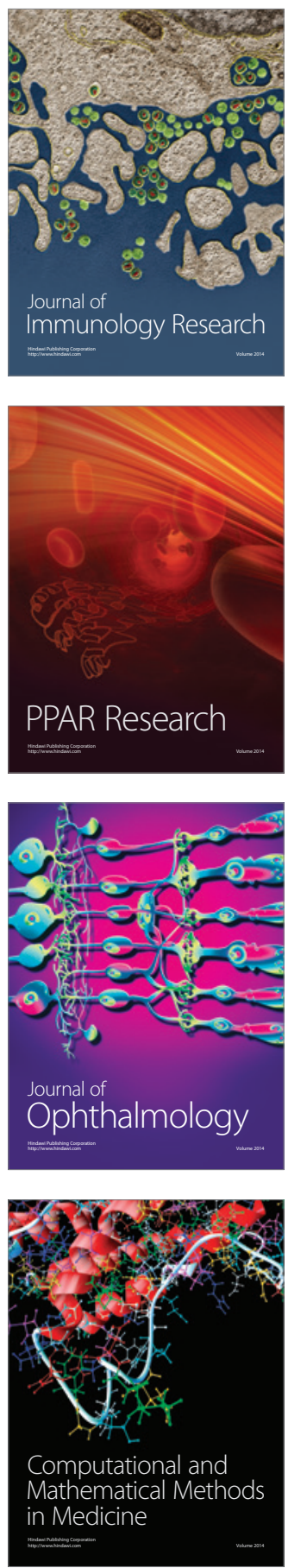

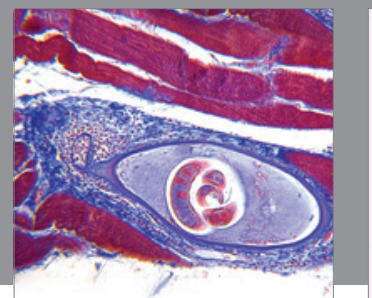

Gastroenterology

Research and Practice
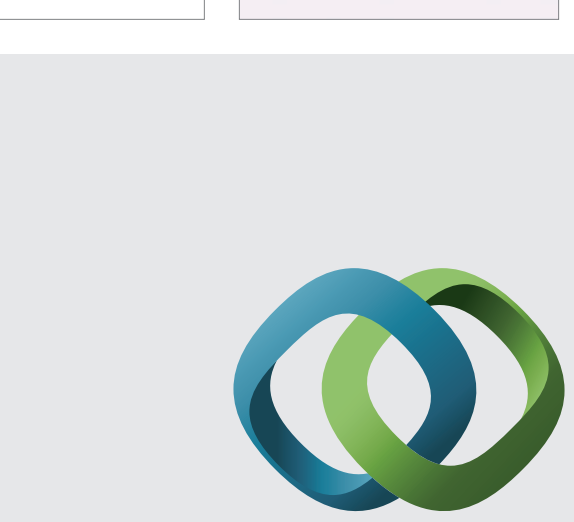

\section{Hindawi}

Submit your manuscripts at

http://www.hindawi.com
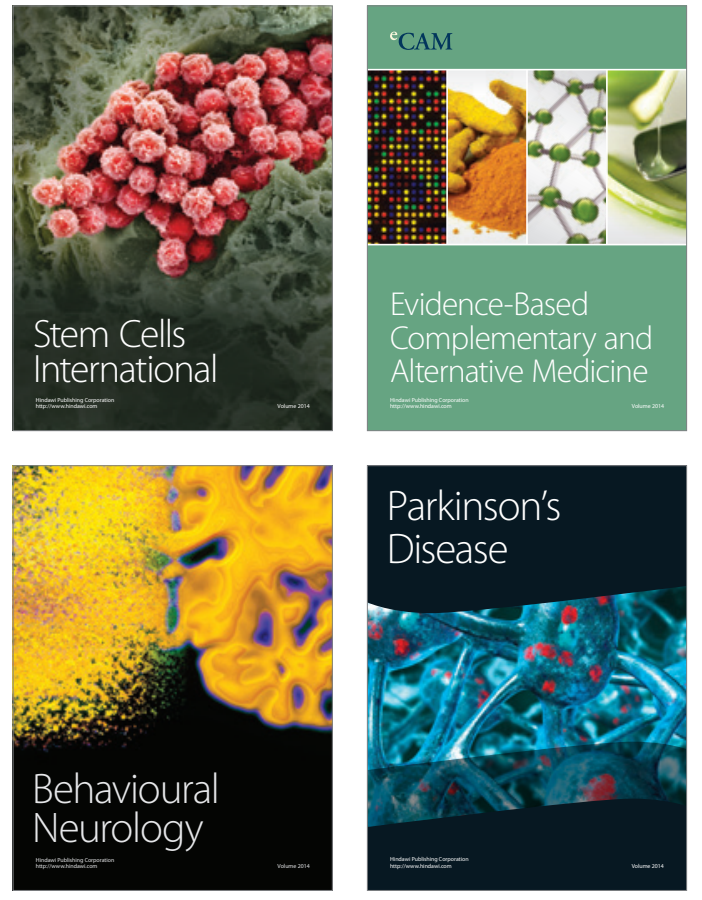
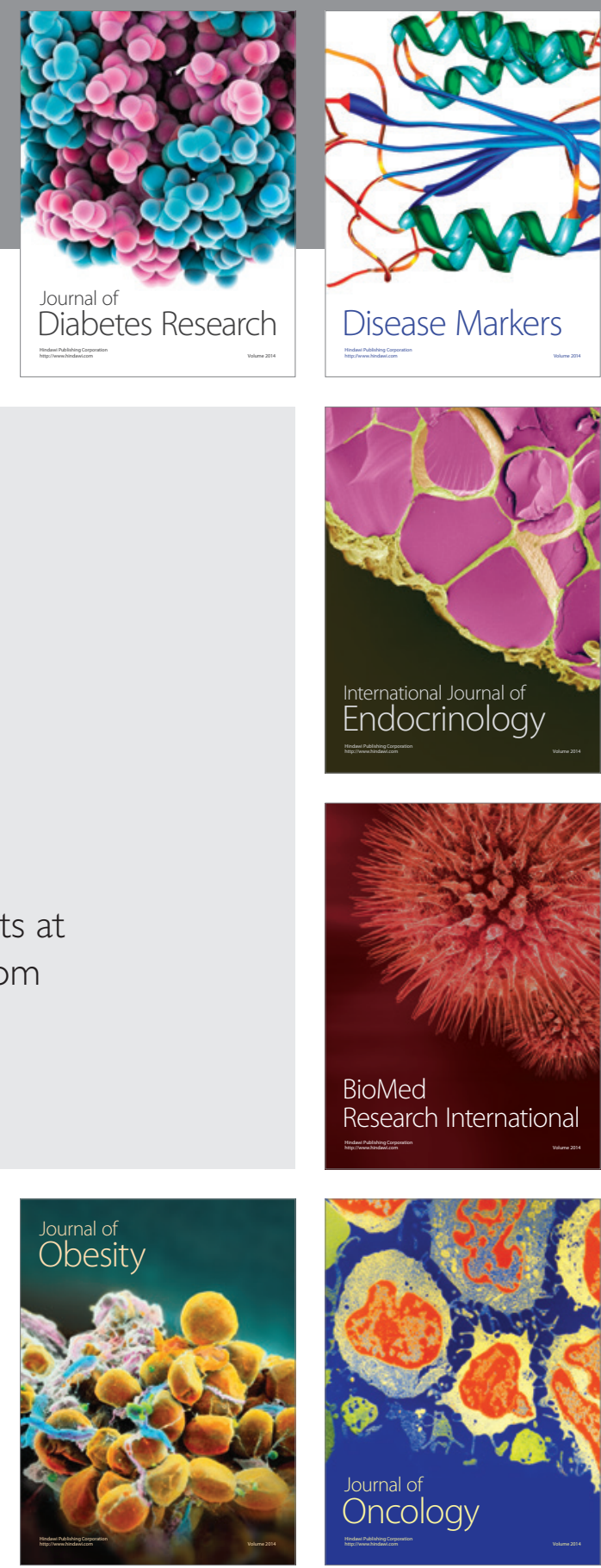

Disease Markers
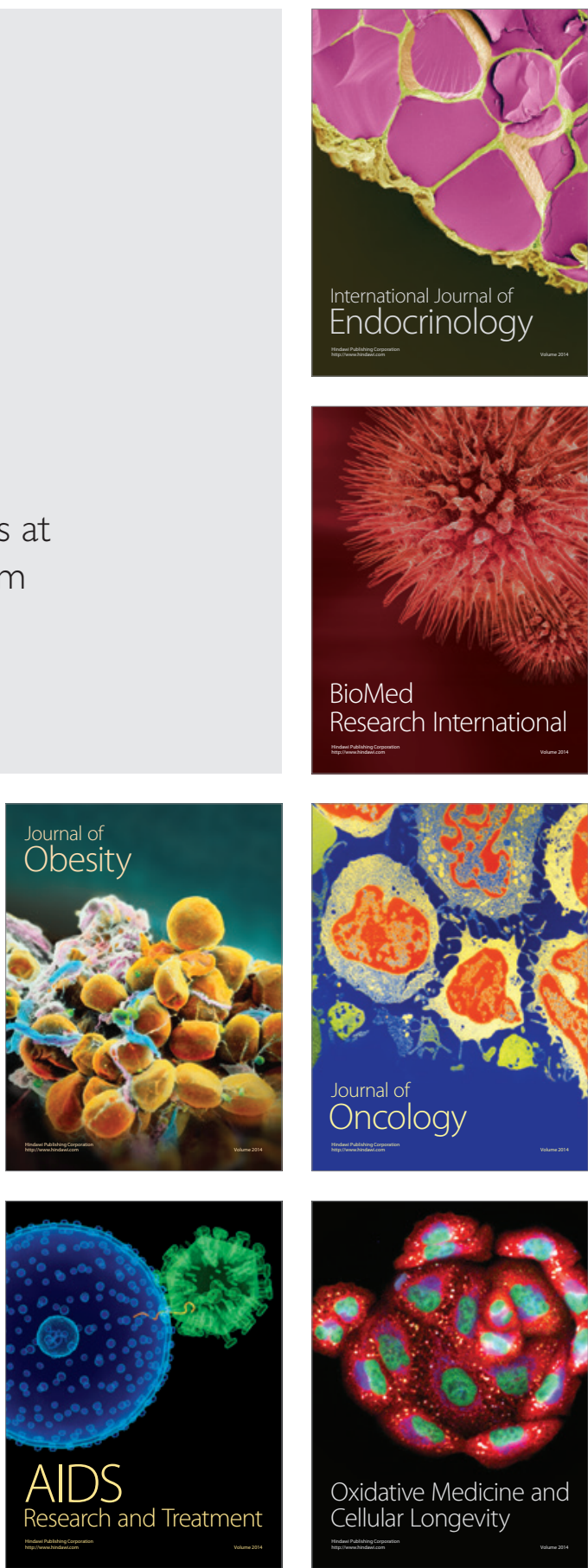